\title{
Quantum Tunneling in Deformed Quantum Mechanics with Minimal Length
}

\author{
Xiaobo Guo, ${ }^{1}$ Bochen Lv, ${ }^{2}$ Jun Tao, ${ }^{2}$ and Peng Wang ${ }^{2}$ \\ ${ }^{1}$ School of Science, Southwest University of Science and Technology, Mianyang 621010, China \\ ${ }^{2}$ Center for Theoretical Physics, College of Physical Science and Technology, Sichuan University, Chengdu 610064, China
}

Correspondence should be addressed to Peng Wang; pengw@scu.edu.cn

Received 14 September 2016; Revised 16 November 2016; Accepted 5 December 2016

Academic Editor: Elias C. Vagenas

Copyright (C) 2016 Xiaobo Guo et al. This is an open access article distributed under the Creative Commons Attribution License, which permits unrestricted use, distribution, and reproduction in any medium, provided the original work is properly cited. The publication of this article was funded by SCOAP . $^{3}$

In the deformed quantum mechanics with a minimal length, one WKB connection formula through a turning point is derived. We then use it to calculate tunneling rates through potential barriers under the WKB approximation. Finally, the minimal length effects on two examples of quantum tunneling in nuclear and atomic physics are discussed.

\section{Introduction}

Various theories of quantum gravity, such as string theory, loop quantum gravity, and quantum geometry, predict the existence of a minimal length [1-3]. For a review of a minimal length in quantum gravity, see [4]. Some realizations of the minimal length from various scenarios have been proposed. Specifically, one of the most popular models is the Generalized Uncertainty Principle (GUP) [5, 6], derived from the modified fundamental commutation relation:

$$
[X, P]=i \hbar\left(1+\beta P^{2}\right)
$$

where $\beta=\beta_{0} \ell_{p}^{2} / \hbar^{2}=\beta_{0} / c^{2} m_{p l}^{2}, m_{p l}$ is the Planck mass, $\ell_{p}$ is the Planck length, and $\beta_{0}$ is a dimensionless parameter. With this modified commutation relation, one can easily find

$$
\Delta X \Delta P \geq \frac{\hbar}{2}\left[1+\beta(\Delta P)^{2}\right],
$$

which leads to the minimal measurable length:

$$
\Delta X \geq \Delta_{\min }=\hbar \sqrt{\beta}=\sqrt{\beta_{0}} e_{p} .
$$

The GUP has been extensively studied recently; see, for example, [7-14]. For a review of the GUP, see [15].
To study 1D quantum mechanics with the deformed commutators (1), one can exploit the following representation for $X$ and $P$ :

$$
\begin{aligned}
& X=X_{0}, \\
& P=P_{0}\left(1+\frac{\beta}{3} P_{0}^{2}\right),
\end{aligned}
$$

where $\left[X_{0}, P_{0}\right]=i \hbar$. It can easily show that such representation fulfills the relation (1) to $\mathcal{O}(\beta)$. Furthermore, we can adopt the position representation:

$$
\begin{aligned}
X_{0} & =x, \\
P_{0} & =\frac{\hbar}{i} \frac{\partial}{\partial x} .
\end{aligned}
$$

Therefore for a quantum system described by

$$
H=\frac{P^{2}}{2 m}+V(X),
$$

the deformed stationary Schrodinger equation in the position representation is

$$
\frac{d^{2} \psi(x)}{d x^{2}}-\ell_{\beta}^{2} \frac{d^{4} \psi(x)}{d x^{4}}+\frac{2 m[E-V(x)]}{\hbar^{2}} \psi(x)=0,
$$

where $\ell_{\beta}^{2} \equiv(2 / 3) \hbar^{2} \beta$ and terms of order $\beta^{2}$ are neglected. 
If (7) with $\beta=0$ can be solved exactly, one could use the perturbation method to solve (7) by treating the term with $\ell_{\beta}^{2}$ as a small correction. However for the general $V(x)$, one might need other methods to solve (7). In fact, the WKB approximation in deformed space has been considered [16]. In [16], the authors considered the deformed commutation relation

$$
[X, P]=i \hbar f(P),
$$

where $f(P)$ is some function. For $f(P)$, one could solve the differential equation:

$$
\frac{d P(p)}{d p}=f(P)
$$

for $P(p)$, and $p(P)$ denotes the inverse function of $P(p)$. It is interesting to note that there might be more than one inverse function for $P(p)$. However, one usually finds that there is only one inverse function $p(P)$ which vanishes at $P=0$. The remaining ones are called "runaway" solutions, which are not physical and should be discarded $[17,18]$. Then, they used the WKB approximation to show that the solution to the deformed Schrodinger equation:

$$
P^{2}\left(\frac{\hbar}{i} \frac{d}{d x}\right) \psi(x)+2 m[V(x)-E] \psi(x)=0,
$$

was

$$
\begin{aligned}
& \psi(x) \\
& =\frac{1}{\sqrt{|P(x) f(P(x))|}}\left(C_{1} \exp \left[\frac{i}{\hbar} \int^{x} p(P(x)) d x\right]\right. \\
& \left.+C_{2} \exp \left[-\frac{i}{\hbar} \int^{x} p(P(x)) d x\right]\right),
\end{aligned}
$$

where $P(x)=\sqrt{2 m[E-V(x)]}$. Moreover, it also showed that condition,

$$
\left|P^{2}(x)\right| \gg \hbar\left|\frac{d}{d x} P(x) f(P(x))\right|,
$$

had to be satisfied to make the WKB approximation valid. However, condition (12) fails near a turning point, where $P(x)=0$.

For the case with $f(P)=1+\beta P^{2}$, we derived one $\mathrm{WKB}$ connection formula through turning points and BohrSommerfeld quantization rule in [19]. In this paper, we continue to consider other WKB connection formulas and calculate tunneling rates through potential barriers. The remainder of our paper is organized as follows. In Section 2, we derive one WKB connection formula and use it to find the formula for the tunneling rate through a potential barrier. Then two examples of quantum tunneling in nuclear and atomic physics are discussed in Section 3. Section 4 is devoted to our conclusions.

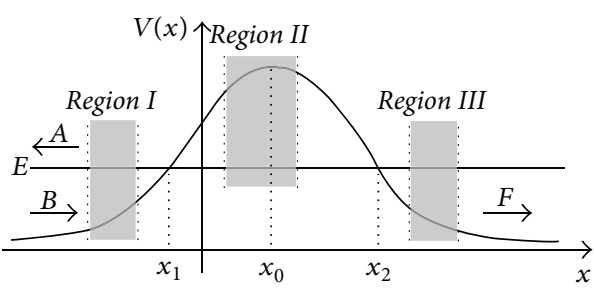

FIGURE 1: Scattering from a potential barrier.

\section{Tunneling through Potential Barriers}

We now consider WKB description of tunneling through a potential barrier $V(x)$, which vanishes as $x \rightarrow \pm \infty$ and rises monotonically to its maximum $V_{\max }$ at $x=x_{0}$ as $x$ approaches $x_{0}$ from either the left or the right side of $x_{0}$. In Figure 1, we plot the potential $V(x)$. For a particle of energy $E$, there are two turning points $x=x_{1}$ and $x=x_{2}, x_{1}<x_{2}$, at which $V(x)=E$. There are two classical allowed regions, Region I with $x<x_{1}$ and Region III with $x>x_{2}$. To describe tunneling, we need to choose appropriate boundary conditions in the classical allowed regions. We postulate an incident right-moving wave in Region I, where the WKB approximation solution to (10) includes a wave incident, the barrier and a reflected wave:

$$
\begin{aligned}
\psi(x)= & \frac{A \exp \left[-(i / \hbar) \int_{x}^{x_{1}} p(P(x)) d x\right]}{\sqrt{|P(x) f(P(x))|}} \\
& +\frac{B \exp \left[(i / \hbar) \int_{x}^{x_{1}} p(P(x)) d x\right]}{\sqrt{|P(x) f(P(x))|}} .
\end{aligned}
$$

In Region III, there is only a transmitted wave:

$$
\psi(x)=\frac{F \exp \left[(i / \hbar) \int_{x_{2}}^{x} p(P(x)) d x\right]}{\sqrt{|P(x) f(P(x))|}} .
$$

In the classically forbidden Region II, there are exponentially growing and decaying solutions:

$$
\begin{aligned}
\psi(x)= & \frac{C \exp \left[-(1 / \hbar) \int_{x_{1}}^{x}|p(P(x))| d x\right]}{\sqrt{|P(x) f(P(x))|}} \\
& +\frac{D \exp \left[(1 / \hbar) \int_{x_{1}}^{x}|p(P(x))| d x\right]}{\sqrt{|P(x) f(P(x))|}} \\
= & \frac{C^{\prime} \exp \left[(1 / \hbar) \int_{x}^{x_{2}}|p(P(x))| d x\right]}{\sqrt{|P(x) f(P(x))|}} \\
& +\frac{D^{\prime} \exp \left[-(1 / \hbar) \int_{x}^{x_{2}}|p(P(x))| d x\right]}{\sqrt{|P(x) f(P(x))|}},
\end{aligned}
$$


where

$$
\begin{aligned}
C^{\prime} & =C e^{-\eta}, \\
D^{\prime} & =D e^{\eta}, \\
\eta & \equiv \frac{1}{\hbar} \int_{x_{1}}^{x_{2}}|p(P(x))| d x .
\end{aligned}
$$

To calculate the tunneling rate, we need to use connection formulas to relate $F, C / C^{\prime}$, and $D / D^{\prime}$ to $A$. In [19], we derived one WKB connection formula around $x=x_{1}$ in the case with $f(P)=1+\beta P^{2}$. If $D=0$, we found that

$$
\begin{array}{r}
\frac{C}{\sqrt{|P(x) f(P(x))|}} \exp \left(-\frac{1}{\hbar} \int_{x_{1}}^{x}|p(P(x))| d x\right) \rightarrow \\
\frac{2 C}{\sqrt{|P(x) f(P(x))|}} \sin \left(\frac{1}{\hbar} \int_{x_{1}}^{x} p(P(x)) d x+\frac{\pi}{4}\right), \\
\text { to } \mathcal{O}\left(\beta^{2}\right),
\end{array}
$$

which gives

$$
A=C e^{-i \pi / 4} .
$$

In what follows, we will derive a WKB connection formula around $x=x_{2}$ to relate $C^{\prime}$ and $D^{\prime}$ to $F$ and then calculate the tunneling rate through the potential barrier.

To match WKB solutions, we need to solve the deformed Schrodinger equation (7) in the vicinity of the turning point $x=x_{2}$. A linear approximation to the potential $V(x)$ near the turning point $x=x_{2}$ is

$$
V(x) \approx V\left(x_{2}\right)-\left|V^{\prime}\left(x_{2}\right)\right|\left(x-x_{2}\right),
$$

where $V\left(x_{2}\right)=E$. To simplify (7), a new dimensionless variable $\rho$ could be introduced:

$$
\rho=\left(x_{2}-x\right)\left(\frac{2 m\left|V^{\prime}\left(x_{2}\right)\right|}{\hbar^{2}}\right)^{1 / 3} .
$$

Thus, (7) becomes

$$
-\alpha^{2} \frac{d^{4} \psi(\rho)}{d \rho^{4}}+\frac{d^{2} \psi(\rho)}{d \rho^{2}}-\rho \psi(\rho)=0,
$$

where $\alpha^{2}=\left(2 m\left|V^{\prime}\left(x_{2}\right)\right| / \hbar^{2}\right)^{2 / 3} \ell_{\beta}^{2}$. The differential equation (7) can be solved by Laplace's method [19]. Integral representations of the solutions are

$$
I(\rho)=\int_{C} \exp \left(\rho t+\frac{\alpha^{2} t^{5}}{5}-\frac{t^{3}}{3}\right) d t,
$$

where the contour $C$ is chosen so that the integrand vanishes at endpoints of $C$. Specifically, define five sectors:

$$
\Theta_{n} \equiv\left[\frac{2 n \pi+\pi / 2}{5}, \frac{2 n \pi+3 \pi / 2}{5}\right], \quad n=0,1,2,3,4 .
$$

The contour $C$ must originate at one of them and terminate at another.

The asymptotic expressions of $I(\rho)$ for large values of $\rho$ can be obtained by evaluating the integral (22) using the method of steepest descent. To do this, we make the change of variables $t=|\rho|^{1 / 2} s$ and find

$$
I(\rho)=|\rho|^{1 / 2} \int_{C} \exp \left[|\rho|^{3 / 2} f_{ \pm}(s)\right] d s,
$$

where $f_{ \pm}(s) \equiv \pm s-s^{3} / 3+a s^{5} / 5$ with + for $\rho>0$ and - for $\rho<0$, and $a \equiv \alpha^{2}|\rho| \ll 1$ in the physical region [19]. We will show below that there exists a steepest descent contour ranging from $s=\infty \exp (7 \pi i / 5)$ to $s=\infty \exp (9 \pi i / 5)$, which is the red contour in Figure 3. Such contour could let us match the asymptotic expression of $I(\rho)$ at large negative value of $\rho$ with the WKB solution (14) in Region III. Note that $\infty \exp (7 \pi i / 5) \in \Theta_{3}$ and $\infty \exp (7 \pi i / 5) \in \Theta_{4}$.

The method of steepest descent is very powerful to calculate integrals of the form:

$$
I(x)=\int_{C} g(z) e^{x f(z)} d z,
$$

where $C$ is a contour in the complex plane. We are usually interested in the behavior of $I(x)$ as $x \rightarrow \infty$. The key step of the method of steepest descent is applying Cauchy's theorem to deform the contours $C$ to the contours coinciding with steepest descent paths. Around a saddle point $z_{0}$, where $f^{\prime}\left(z_{0}\right)=0$, there are two constant-phase (steepest) contours, on which $\operatorname{Im} f(z)$ is constant, passing through $z_{0}$ if $f^{\prime \prime}\left(z_{0}\right) \neq$ 0 . One of them is a steepest descent contour, along which $\operatorname{Re} f(z)$ increases as we go towards $z_{0}$. The other is a steepest ascent contour, along which $\operatorname{Re} f(z)$ decreases as we go towards $z_{0}$. If $I(x)$ is integrated along the steepest descent contour, the asymptotic behavior of $I(x)$ is dominated by the contribution from the saddle point $z_{0}$.

In Figures 2 and 3, we plot saddle points (red points in figures) of $f_{+}(s)$ and $f_{-}(s)$, respectively, and constant-phase contours passing through them. Specifically, saddle points of $f_{+}(s)$ are

$$
\begin{aligned}
& s= \pm \lambda_{+} \equiv \pm \frac{\sqrt{1-\sqrt{1-4 a}}}{\sqrt{2 a}}, \\
& s= \pm \eta_{+} \equiv \pm \frac{\sqrt{1+\sqrt{1-4 a}}}{\sqrt{2 a}},
\end{aligned}
$$

and these of $f_{-}(s)$ are

$$
\begin{aligned}
& s= \pm \lambda_{-} \equiv \pm \frac{\sqrt{1-\sqrt{1+4 a}}}{\sqrt{2 a}}, \\
& s= \pm \eta_{-} \equiv \pm \frac{\sqrt{1+\sqrt{1+4 a}}}{\sqrt{2 a}} .
\end{aligned}
$$

The red contours in Figures 2 and 3 are the steepest descent contours connecting $s=\infty \exp (7 \pi i / 5)$ to $s=\infty \exp (9 \pi i / 5)$, 


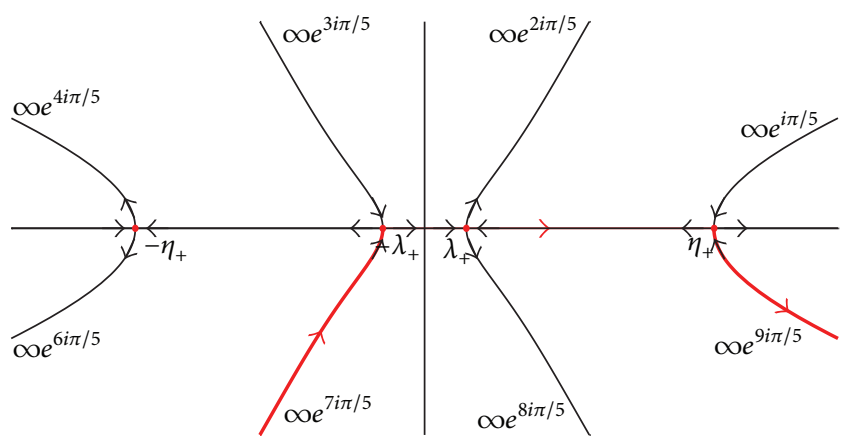

FIGURE 2: Saddle points and constant-phase (steepest) contours of $f_{+}(s)$. The red contour is a steepest descent contour, along which $I(\rho)$ is integrated. The black arrows on the constant-phase contours around saddle points denote the directions in which values of $\operatorname{Re} f_{+}(s)$ increase.

along which the integral (24) is integrated. Note that red arrows on them denote the steepest contours' directions. On the other hand, the black arrows on the constant-phase contours around saddle points denote the directions in which values of $\operatorname{Re} f_{ \pm}(s)$ increase. Following the black arrows on the red contour in Figure 2, we find that $\operatorname{Re} f_{+}\left(-\lambda_{+}\right)$and $\operatorname{Re} f_{+}\left(\eta_{+}\right)$are smaller than $\operatorname{Re} f_{+}\left(\lambda_{+}\right)$. Thus for the case with $\rho>0$, the asymptotic expression of $I(\rho)$ is dominated by the contribution from the saddle $s=\lambda_{+}$. The method of steepest descent gives

$$
\begin{aligned}
I(1 & \left.\ll \rho \ll \alpha^{-2}\right) \\
& \sim \frac{\sqrt{\pi} \exp \left[\rho^{3 / 2} f_{+}\left(\lambda_{+}\right)\right]}{\rho^{1 / 4}} \sqrt{\frac{2}{\left|f_{+}^{\prime \prime}\left(\lambda_{+}\right)\right|}} \\
& \sim \frac{\sqrt{\pi}(1+(3 / 4) a)}{\rho^{1 / 4}} \exp \left[\frac{2 \rho^{3 / 2}}{3}\left(1+\frac{3 a}{10}\right)\right],
\end{aligned}
$$

where $a \ll 1$ is used, and terms of $\mathcal{O}\left(a^{2}\right)$ are neglected in the second line. For the case with $\rho>0$, Figure 3 shows that the asymptotic expression of $I(\rho)$ is dominated by the contribution from the saddle $s=-\lambda_{-}$, and hence we find

$$
\begin{aligned}
& I\left(-1 \gg \rho \gg-\alpha^{-2}\right) \\
& \sim \frac{\sqrt{\pi} \exp ((\pi / 4) i) \exp \left[|\rho|^{3 / 2} f_{-}\left(-\lambda_{-}\right)\right]}{|\rho|^{1 / 4}} \\
& \cdot \sqrt{\frac{2}{\left|f_{-}^{\prime \prime}\left(-\lambda_{+}\right)\right|}} \sim \frac{\sqrt{\pi} \exp ((\pi / 4) i)(1-(3 / 4) a)}{|\rho|^{1 / 4}} \\
& \cdot \exp \left[\frac{2 i|\rho|^{3 / 2}}{3}\left(1-\frac{3 a}{10}\right)\right],
\end{aligned}
$$

where terms of $\mathcal{O}\left(a^{2}\right)$ are neglected in the second line.

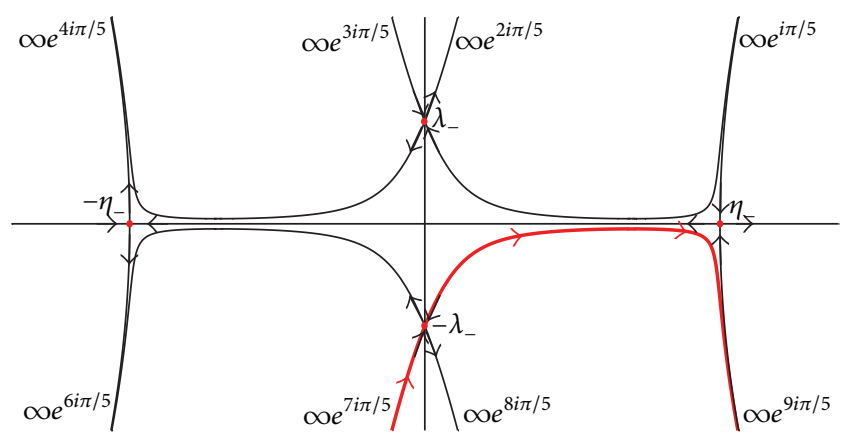

FIgURE 3: Saddle points and constant-phase (steepest) contours of $f_{-}(s)$. The red contour is a steepest descent contour, along which $I(\rho)$ is integrated. The black arrows on the constant-phase contours around saddle points denote the directions in which values of $\operatorname{Re} f_{-}(s)$ increase.

Around the turning point $x=x_{2},\left|x-x_{2}\right| \ll 1$, and $P(x) \sim \sqrt{2 m\left|V^{\prime}\left(x_{2}\right)\right|} \sqrt{x-x_{2}}$. In this region, we find that WKB solutions (14) and (15) become

$$
\begin{aligned}
\psi(x) & \sim \frac{F}{\left(2 m\left|V^{\prime}\left(x_{2}\right)\right| / \hbar^{2}\right)^{1 / 3} \hbar} \frac{1-3 a / 4}{|\rho|^{1 / 4}} \\
\cdot & \exp \left[\frac{2 i|\rho|^{3 / 2}}{3}\left(1-\frac{3 a}{10}\right)\right], \\
\psi(x) & \sim \frac{1}{\left(2 m\left|V^{\prime}\left(x_{2}\right)\right| / \hbar^{2}\right)^{1 / 3} \hbar} \\
\cdot & \frac{1+(3 / 4) a}{\rho^{1 / 4}}\left\{C^{\prime} \exp \left[\frac{2 \rho^{3 / 2}}{3}\left(1+\frac{3 a}{10}\right)\right]\right. \\
+ & \left.D^{\prime} \exp \left[-\frac{2 \rho^{3 / 2}}{3}\left(1+\frac{3 a}{10}\right)\right]\right\},
\end{aligned}
$$

where we use $p(P) \approx P-(\beta / 3) P^{3}$ and terms of $\mathcal{O}\left(a^{2}\right)$ are neglected, and we express $x$ in terms of $\rho$ using (20). In the overlap regions, where $|\rho| \gg 1$ and $\left|x-x_{2}\right| \ll 1$, matching WKB solutions (30) with the $I(\rho)$ 's asymptotic expressions (28) and (29) gives

$$
\begin{aligned}
& C^{\prime}=F \exp \left(-\frac{\pi}{4} i\right), \\
& D^{\prime}=0,
\end{aligned}
$$

which by (16) lead to $C=F \exp (-(\pi / 4) i) e^{\eta}$ and $D=0$. Since $D=0,(18)$ gives

$$
F=i A e^{-\eta},
$$

and the transmission probability is

$$
T=\frac{|F|^{2}}{|A|^{2}} \sim e^{-2 \eta}
$$




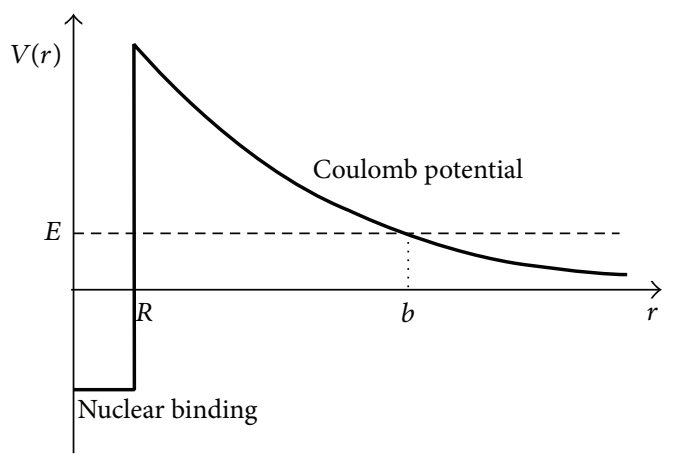

FIGURE 4: The potential energy of an $\alpha$-particle in a radioactive nucleus.

\section{Examples}

The dimensionless number $\beta_{0}=c^{2} m_{p l}^{2} \beta=\hbar^{2} \beta / \ell_{p}^{2}$ plays an important role when implications and applications of nonzero minimal length are discussed. Normally, if the minimal length is assumed to be order of the Planck length $\ell_{p}$, one has $\beta_{0} \sim 1$. In [9], based on the precision measurement of STM current, an upper bound of $\beta_{0}$ was given by $\beta_{0}<10^{21}$. In the following, we use (33) to study effects of GUP on $\alpha$ decay and cold electrons emission from metal via strong external electric field.

3.1. $\alpha$ Decay. The decay of a nucleus into an $\alpha$-particle (charge $2 e$ ) and a daughter nucleus (charge $Z e$ ) can be described as the tunneling of an $\alpha$-particle through a barrier caused by the Coulomb potential between the daughter and the $\alpha$-particle (Figure 4) [20]. For an $\alpha$-particle of energy $E$ in the potential in Figure 4, there are two turning points, the nuclear radius $R$ and the outer turning point $b$, which is determined by

$$
\begin{aligned}
& E=\frac{Z e^{2}}{2 \pi \varepsilon_{0} r} \Longrightarrow \\
& b=\frac{Z e^{2}}{2 \pi \varepsilon_{0} E} .
\end{aligned}
$$

The exponent $\eta$ in (33) is

$$
\begin{aligned}
\eta & =\frac{1}{\hbar} \int_{R}^{b}\left|p\left(\sqrt{2 m E} \sqrt{1-\frac{Z_{1} Z_{2} e^{2}}{4 \pi \varepsilon_{0} E r}}\right)\right| d x \\
& \approx \frac{\sqrt{2 m E}}{\hbar}\left\{b \arccos \left(\sqrt{\frac{R}{b}}\right)-\sqrt{R(b-R)}\right. \\
& +\frac{2 m E \beta}{3}\left[3 b \operatorname{arcsec} \sqrt{\frac{b}{R}-\sqrt{R(b-R)}}\right. \\
& \left.\left.-2 b \sqrt{\frac{b-R}{R}}\right]\right\}
\end{aligned}
$$

where $m$ is the mass of the $\alpha$-particle. At low energies (relative to the height of the Coulomb barrier at $r=R$ ), we have $b \gg R$ and then

$$
\eta \approx \frac{\sqrt{2 m} e^{2}}{4 \varepsilon_{0} \hbar}\left[1-\frac{8 m E \beta}{3 \pi} \sqrt{\frac{b}{R}}\right] \frac{Z}{\sqrt{E}} .
$$

The probability of emission of an $\alpha$-particle is proportional to $e^{-2 \eta}$ and hence the lifetime of the parent nucleus is about

$$
\tau \sim e^{2 \eta} .
$$

The density of nuclear matter is relatively constant, so $R^{3}$ is proportional to the number of nucleons $A$. Empirically, we have

$$
R \sim A^{1 / 3} f m
$$

Therefore, we find

$$
\begin{aligned}
& \ln \tau^{-1} \approx \text { const } \\
& -\frac{\sqrt{2 m} e^{2}}{2 \varepsilon_{0} \hbar}\left[1-\beta_{0}\left(\frac{E}{\mathrm{MeV}}\right)^{1 / 2} \sqrt{Z A^{-1 / 3}} \times 10^{-40}\right] \\
& \cdot \frac{Z}{\sqrt{E}} .
\end{aligned}
$$

On the other hand, a large collection of data shows that a good fit to the lifetime data obeys the Geiger-Nuttall law [21]

$$
\ln \tau^{-1}=C_{1}-C_{2} \frac{Z}{\sqrt{E}}
$$

where $C_{1}$ and $C_{2}$ are constants. If the effects of GUP do not make (39) differ too much from the Geiger-Nuttall law, it will put an upper bound:

$$
\beta_{0}<10^{40} .
$$

The GUP correction to the $\alpha$-decay has also been considered in [22]. We both find that the effects of the GUP would increase the tunneling probability and hence decrease the lifetime $\tau$.

3.2. Electron Emission from the Surface of Cold Metals. If a metal is placed in a very strong electric field, then there exists cold emission of electrons from the surface of the metal. This emission of the electrons can be explained via quantum tunneling. In [23], the shape of a tunneling barrier was assumed to be the exact triangular barrier, which has been known as the Fowler-Nordheim tunneling. Note that work must be done to remove an electron from the surface of a metal. In "free electron gas" model, one could hence take the potential energy of the electron inside the metal to be zero and for the outside to be $V(x)=V_{0}>0$. At the absolute zero temperature, if the Fermi energy of these electrons $E_{F}$ is less than $V_{0}$, therefore after reaching the surface of the metal, they are reflected back into the metal. Now if the external electric 


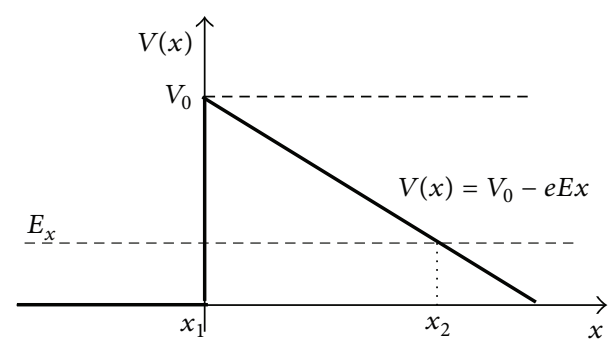

FIGURE 5: The potential energy inside and outside a metallic surface when an external electric field $E$ is added.

field is applied toward the surface of the metal, the potential energy becomes

$$
V(x)= \begin{cases}V_{0}-e E x & \text { for } x>0 \\ 0 & \text { for } x<0\end{cases}
$$

where $E$ is the magnitude of the electric field. This potential is shown in Figure 5.

We now use (33) to calculate the GUP modified transmission probability. For an electron of energy $E_{x} \leq E_{F}<V_{0}$, there are two turning points:

$$
\begin{aligned}
& x_{1}=0, \\
& x_{2}=\frac{V_{0}-E_{x}}{e E} .
\end{aligned}
$$

The exponent $\eta$ in (33) is

$$
\begin{aligned}
\eta\left(E_{x}\right) & \equiv \frac{1}{\hbar} \int_{x_{1}}^{x_{2}}\left|p\left(\sqrt{2 m\left[E_{x}-V_{0}+e E x\right]}\right)\right| d x \\
& \approx \frac{2 \sqrt{2 m}\left(V_{0}-E_{x}\right)^{3 / 2}}{3 e E \hbar}\left[1-\frac{2 m \beta\left(V_{0}-E_{x}\right)}{5}\right],
\end{aligned}
$$

which gives the transmission probability $T\left(E_{x}\right) \approx e^{-2 \eta\left(E_{x}\right)}$.

Next we want to calculate the electric current density in this case. As a consequence of the GUP, the number of quantum states should be changed to [24]

$$
\frac{V d^{3} p}{(2 \pi \hbar)^{3}\left(1+\beta p^{2}\right)^{3}}
$$

where $p^{2}=p_{i} p^{i}$. Therefore, the electric current density is given by

$$
J=e \int \frac{2 d p_{x} d p_{y} d p_{z}}{(2 \pi \hbar)^{3}\left(1+\beta p^{2}\right)^{3}} \frac{p_{x}}{m} T\left(E_{x}\right)
$$

where $E_{x}=p_{x}^{2} / 2 m$. The range of variations of $p_{x}, p_{y}$, and $p_{z}$ is limited to the points inside the Fermi sphere

$$
p_{x}^{2}+p_{y}^{2}+p_{z}^{2} \leq 2 m E_{F}
$$

To calculate $J$, we use cylindrical coordinates,

$$
\begin{aligned}
p_{y} & =\rho \cos \phi, \\
p_{z} & =\rho \sin \phi, \\
\rho^{2}+p_{x}^{2} & \leq 2 m E_{F},
\end{aligned}
$$

and have

$$
\begin{aligned}
J= & \frac{4 \pi e}{(2 \pi \hbar)^{3}} \int_{0}^{\sqrt{2 m E_{F}}} \frac{p_{x}}{m} T\left(E_{x}\right) d p_{x} \\
& \cdot \int_{0}^{\sqrt{2 m E_{F}-p_{x}^{2}}} \frac{\rho d \rho}{\left[1+\beta\left(\rho^{2}+p_{x}^{2}\right)\right]^{3}} \approx \frac{2 \pi e}{(2 \pi \hbar)^{3}} \\
& \cdot \int_{0}^{\sqrt{2 m E_{F}}} \frac{p_{x}}{m} T\left(E_{x}\right)\left(2 m E_{F}-p_{x}^{2}\right) \\
& \cdot\left[1-\frac{3 \beta}{2}\left(2 m E_{F}+p_{x}^{2}\right)\right] d p_{x} .
\end{aligned}
$$

To simplify the result, we change $E_{x}$ to

$$
\epsilon=E_{F}-E_{x} \text {. }
$$

Therefore, one has

$$
J \approx \frac{e m}{2 \pi^{2} \hbar^{3}} \int_{0}^{E_{F}} \epsilon T(\epsilon)\left[1-3 \beta m\left(2 E_{F}-\epsilon\right)\right] d \epsilon .
$$

Since $T(\epsilon)$ decreases rapidly with increasing $\epsilon$, therefore in $T(\epsilon)$, we can expand $\left(V_{0}-E_{F}+\epsilon\right)^{3 / 2}$ :

$$
\left(V_{0}-E_{F}+\epsilon\right)^{3 / 2} \approx\left(V_{0}-E_{F}\right)^{3 / 2}+\frac{3}{2} \epsilon\left(V_{0}-E_{F}\right)^{1 / 2} .
$$

We find

$$
\begin{aligned}
J \approx & \frac{e m}{2 \pi^{2} \hbar^{3}} \\
& \cdot \exp \left[-\frac{2 Q}{3}\right] \frac{\left(V_{0}-E_{F}\right)^{2}}{Q^{2}}\left[1+\frac{4 \beta m\left(V_{0}-E_{F}\right) Q}{15}\right],
\end{aligned}
$$

where we extend the range of integration in $(51)$ to $(0, \infty)$, and

$$
Q=\frac{2 \sqrt{2 m}\left(V_{0}-E_{F}\right)^{3 / 2}}{\hbar e E} .
$$

In [25], the Fowler-Nordheim tunneling in device grade ultra-thin 3-6 $\mathrm{nm} n^{+}$poly-Si/ $/ \mathrm{SiO}_{2} / n$-Si structures has been analyzed. Typically for this electron tunneling, we have

$$
\begin{aligned}
Q & \sim 10, \\
V_{0}-E_{F} & \sim 1 \mathrm{eV}, \\
m & \sim 0.5 \mathrm{MeV} .
\end{aligned}
$$

Therefore from (53), the correction due to GUP is given by

$$
\frac{\delta J}{J} \sim 10^{-50} \beta_{0} .
$$


The comparison of the calculated and experimental tunnel current was plotted in FIG. 8 of [25], which implies $\delta J / J \lesssim$ $10^{-2}$. Then the upper bound on $\beta_{0}$ follows:

$$
\beta_{0}<10^{48} \text {. }
$$

\section{Conclusions}

In this paper, we considered quantum tunneling in the deformed quantum mechanics with a minimal length. We first found one $\mathrm{WKB}$ connection formula through a turning point. Then the tunneling rates through potential barriers were derived using the WKB approximation. Finally, effects of the minimal length on quantum tunneling were discussed in two examples in nuclear and atomic physics, $\alpha$ decay and the Fowler-Nordheim tunneling. Upper bounds on $\beta_{0}$ were given in these two examples, in which one had $\beta_{0}<10^{40}$ and $\beta_{0}<10^{48}$, respectively.

In [9], the effects of the minimal length on electrons tunneling through a potential barrier of form

$$
V(x)=V_{0}[\theta(x)-\theta(x-a)]
$$

were considered. For electrons of the mass $m$ and energy $E<$ $V_{0}$, the GUP deformed Schrodinger equation was solved for the transmission coefficient, which was given by

$$
\frac{T}{T_{0}}=1+\frac{4 m \beta\left(2 E-V_{0}\right)^{2}}{3 V_{0}}+\frac{2 \beta a}{3 \hbar}\left[2 m\left(V_{0}-E\right)\right]^{3 / 2},
$$

where $T_{0}$ is the standard tunneling amplitude and $\beta$ used in [9] is $\beta / 3$ in this paper. Although the exact result for the transmission coefficient was given by (59), it is a good exercise to use (33) to calculate the transmission coefficient and compare the WKB approximated result with the exact one. In fact, there are two turning points $x=0$ and $a$ for potential (58). Thus, we have

$$
T \sim \exp \left[-\frac{2}{\hbar} \int_{0}^{a}\left|p\left(\sqrt{2 m\left(E-V_{0}\right)}\right)\right| d x\right] .
$$

Since $p(P) \approx P-(\beta / 3) P^{3}$, one finds

$$
\frac{T}{T_{0}}=1+\frac{2 \beta a}{3 \hbar}\left[2 m\left(V_{0}-E\right)\right]^{3 / 2},
$$

where $T_{0}$ is the standard WKB tunneling amplitude. It is noteworthy that the second term in the right-hand side of (59) is $\mathcal{O}\left(\hbar^{0}\right)$, while the third term is $\mathcal{O}\left(\hbar^{-1}\right)$ and hence is the leading order term of the WKB expansion. So as expected, the WKB result (61) agrees with the exact one (59) to $\mathcal{O}\left(\hbar^{-1}\right)$. To put an upper bound on $\beta_{0}$, the authors considered the following approximate values:

$$
\begin{aligned}
m & \sim 0.5 \mathrm{MeV} / c^{2}, \\
a & \sim 10^{-10} \mathrm{~m}, \\
E & \sim 10 \mathrm{eV}, \\
V_{0} & \sim 10 \mathrm{eV} .
\end{aligned}
$$

In this case, we find from (59) that

$$
\frac{\delta T}{T_{0}} \sim 10^{-48} \beta_{0}+10^{-51}\left(\frac{V_{0}-E}{1 \mathrm{eV}}\right)^{3 / 2} \beta_{0},
$$

which shows that when $V_{0}-E \gtrsim 6 \mathrm{eV}$, the leading WKB order term dominates, and hence the $\mathrm{WKB}$ approximation is a good one. Note that it was assumed that $V_{0} \approx E$ in [9], and then the first term in the right-hand side of (63) dominates. However, if the leading WKB order term dominates, one can follow the argument in [9] and has

$$
\beta_{0}<\left(\frac{V_{0}-E}{1 \mathrm{eV}}\right)^{-3 / 2} \times 10^{24},
$$

which might put a stronger bound on $\beta_{0}$ in some other case.

In the framework of the doubly special relativity, it has been shown that there is an upper bound on the momentum for a test particle [26]. To incorporate the existence of the minimal measurable length and the maximal measurable momentum, a new form of the GUP has been proposed $[11,27]$ :

$$
[X, P]=i \hbar\left(1-\alpha P+2 \alpha^{2} P^{2}\right),
$$

where $\alpha \equiv \alpha_{0} / m_{p l} c$ is the GUP parameter and can be related to $\beta$ through dimensional analysis with $\left[\alpha^{2}\right]=[\beta]$. Note that the relation $\left[\alpha^{2}\right]=[\beta]$ implies that $\beta_{0} \sim \alpha_{0}^{2}$ since $\beta_{0}=\beta / m_{p l}^{2} c^{2}$. The Hilbert space representation of quantum mechanics with the deformed fundamental commutation relation (65) was investigated in [28]. Comparing to the quantum mechanics with only a minimal measurable length [6], the authors of [28] found that there were several novel and interesting features in this deformed quantum mechanics. For example, it showed that the expectation value of energy in the formal position eigenvectors and the energy at the short wavelength of a test particle were no longer divergent, while both of them were divergent in the quantum mechanics with only a minimal measurable length. Moreover, the authors also found that the position operator $X$ was symmetric but not self-adjoint, while the momentum operator $P$ was self-adjoint due to absence of the minimal uncertainty in momentum.

On the phenomenological side, the upper bounds on $\alpha_{0}$ have been studied [29, 30]. For example, the effects of the minimal length and the maximal momentum on the transition rate of ultra-cold neutrons bouncing above a mirror in the Earth's gravitational field were investigated in [29], and the accuracy of Nesvizhevsky experiments put an upper bound on $\alpha_{0}$ :

$$
\alpha_{0} \leq 10^{29}
$$

which implies that $\beta_{0} \sim \alpha_{0}^{2} \leq 10^{58}$. Moreover, some tighter bounds came from studying the Planck scale effects on current experiments in Superconductivity and muon experiments [30], which gave $\alpha_{0} \leq 10^{17}\left(\beta_{0} \leq 10^{34}\right)$ and $\alpha_{0} \leq 10^{8}\left(\beta_{0} \leq 10^{16}\right)$, respectively.

\section{Competing Interests}

The authors declare that there is no conflict of interests regarding the publication of this paper. 


\section{Acknowledgments}

The authors are grateful to Houwen Wu and Zheng Sun for useful discussions. This work is supported in part by NSFC (Grants nos. 11005016, 11175039, and 11375121).

\section{References}

[1] P. K. Townsend, "Small-scale structure of spacetime as the origin of the gravitational constant," Physical Review D, vol. 15, no. 10, pp. 2795-2801, 1977.

[2] D. Amati, M. Ciafaloni, and G. Veneziano, "Can spacetime be probed below the string size?" Physics Letters B, vol. 216, no. 1-2, pp. 41-47, 1989.

[3] K. Konishi, G. Paffuti, and P. Provero, "Minimum physical length and the generalized uncertainty principle in string theory," Physics Letters. B, vol. 234, no. 3, pp. 276-284, 1990.

[4] L. J. Garay, “Quantum gravity and minimum length," International Journal of Modern Physics A, vol. 10, no. 2, pp. 145-165, 1995.

[5] M. Maggiore, "The algebraic structure of the generalized uncertainty principle," Physics Letters B, vol. 319, no. 1-3, pp. 83-86, 1993.

[6] A. Kempf, G. Mangano, and R. B. Mann, "Hilbert space representation of the minimal length uncertainty relation," Physical Review D, vol. 52, no. 2, pp. 1108-1118, 1995.

[7] L. N. Chang, D. Minic, N. Okamura, and T. Takeuchi, "Effect of the minimal length uncertainty relation on the density of states and the cosmological constant problem," Physical Review D, vol. 65, no. 12, Article ID 125028, 2002.

[8] F. Brau, "Minimal length uncertainty relation and the hydrogen atom," Journal of Physics A: Mathematical and General, vol. 32, no. 44, pp. 7691-7696, 1999.

[9] S. Das and E. C. Vagenas, "Universality of quantum gravity corrections," Physical Review Letters, vol. 101, no. 22, Article ID 221301, 2008.

[10] S. Hossenfelder, M. Bleicher, S. Hofmann, J. Ruppert, S. Scherer, and H. Stöcker, "Signatures in the Planck regime," Physics Letters $B$, vol. 575, no. 1-2, pp. 85-99, 2003.

[11] A. F. Ali, S. Das, and E. C. Vagenas, "Discreteness of space from the generalized uncertainty principle," Physics Letters B, vol. 678, no. 5, pp. 497-499, 2009.

[12] L. Xiang, "Black hole entropy without brick walls," Physics Letters B, vol. 540, no. 1-2, pp. 9-13, 2002.

[13] F. Brau and F. Buisseret, "Minimal length uncertainty relation and gravitational quantum well," Physical Review D, vol. 74, no. 3, Article ID 036002, 2006.

[14] P. Wang, H. Yang, and S. Ying, "Minimal length effects on entanglement entropy of spherically symmetric black holes in the brick wall model," Classical and Quantum Gravity, vol. 33, no. 2, Article ID 025007, 2016.

[15] S. Hossenfelder, "Minimal length scale scenarios for quantum gravity," Living Reviews in Relativity, vol. 16, article 2, 2013.

[16] T. V. Fityo, I. O. Vakarchuk, and V. M. Tkachuk, "WKB approximation in deformed space with minimal length," Journal of Physics A: Mathematical and General, vol. 39, no. 2, pp. 379387, 2006.

[17] J. Z. Simon, "Higher-derivative Lagrangians, nonlocality, problems, and solutions," Physical Review D, vol. 41, no. 12, pp. 37203733, 1990.
[18] B. Mu, P. Wang, and H. Yang, "Thermodynamics and luminosities of rainbow black holes," Journal of Cosmology and Astroparticle Physics, vol. 2015, no. 11, article 045, 2015.

[19] J. Tao, P. Wang, and H. Yang, "Homogeneous field and WKB approximation in deformed quantum mechanics with minimal length," Advances in High Energy Physics, vol. 2015, Article ID 718359, 15 pages, 2015.

[20] G. Gamow, “Zur Quantentheorie des Atomkernes," Zeitschrift für Physik, vol. 51, no. 3-4, pp. 204-212, 1928.

[21] H. Geiger and J. M. Nuttall, "The ranges of the $\alpha$ particles from various radioactive substances and a relation between range and period of transformation," Philosophical Magazine, vol. 22, no. 130, p. 613, 1911.

[22] G. Blado, T. Prescott, J. Jennings, J. Ceyanes, and R. Sepulveda, "Effects of the generalised uncertainty principle on quantum tunnelling," European Journal of Physics, vol. 37, no. 2, Article ID 025401, 2016.

[23] R. H. Fowler and L. Nordheim, "Electron emission in intense electric fields," Proceedings of the Royal Society of London A: Mathematical, Physical and Engineering Sciences, vol. 119, no. 781, pp. 173-181, 1928.

[24] P. Wang, H. Yang, and X. Zhang, "Quantum gravity effects on statistics and compact star configurations," Journal of High Energy Physics, vol. 2010, no. 8, article no. 43, 2010.

[25] M. Depas, B. Vermeire, P. W. Mertens, R. L. Van Meirhaeghe, and M. M. Heyns, "Determination of tunnelling parameters in ultra-thin oxide layer poly-Si/SiO $/$ /Si structures," Solid State Electronics, vol. 38, no. 8, pp. 1465-1471, 1995.

[26] J. L. Cortes and J. Gamboa, "Quantum uncertainty in doubly special relativity," Physical Review D, vol. 71, Article ID 065015, 2005.

[27] A. F. Ali, S. Das, and E. C. Vagenas, "Proposal for testing quantum gravity in the lab," Physical Review D, vol. 84, no. 4, Article ID 044013, 2011.

[28] K. Nozari and A. Etemadi, "Minimal length, maximal momentum, and Hilbert space representation of quantum mechanics," Physical Review D, vol. 85, no. 10, Article ID 104029, 2012.

[29] P. Pedram, K. Nozari, and S. H. Taheri, "The effects of minimal length and maximal mo mentum on the transition rate of ultra cold neutrons in gravitational field," Journal of High Energy Physics, vol. 2011, article 93, 2011.

[30] S. Das and R. B. Mann, "Planck scale effects on some low energy quantum phenomena," Physics Letters B, vol. 704, no. 5, pp. 596599, 2011. 

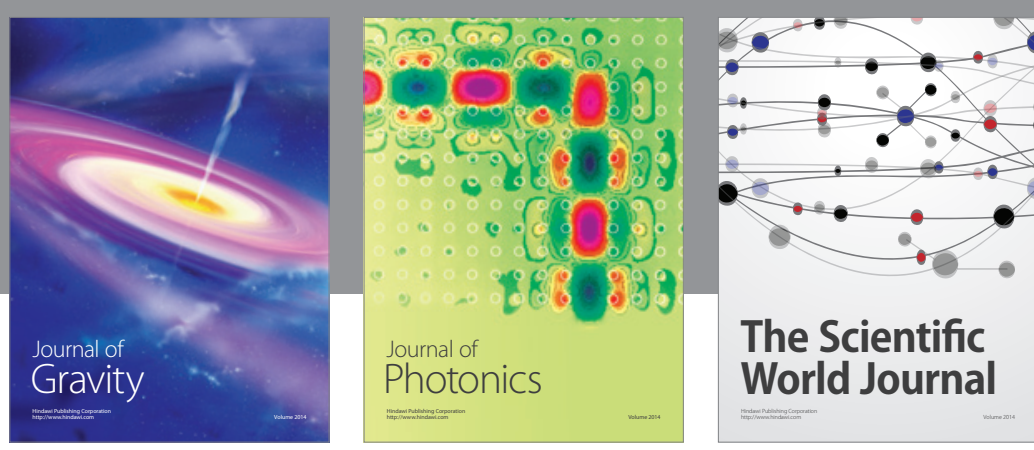

The Scientific World Journal
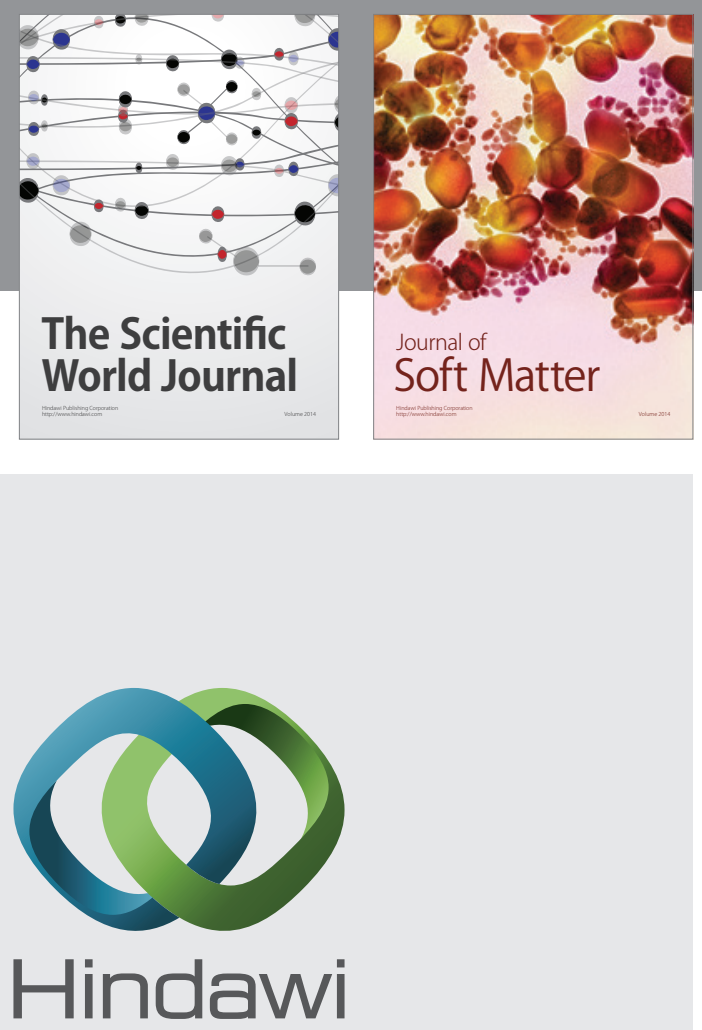

Submit your manuscripts at

http://www.hindawi.com

nternational Journal of

Statistical Mechanics
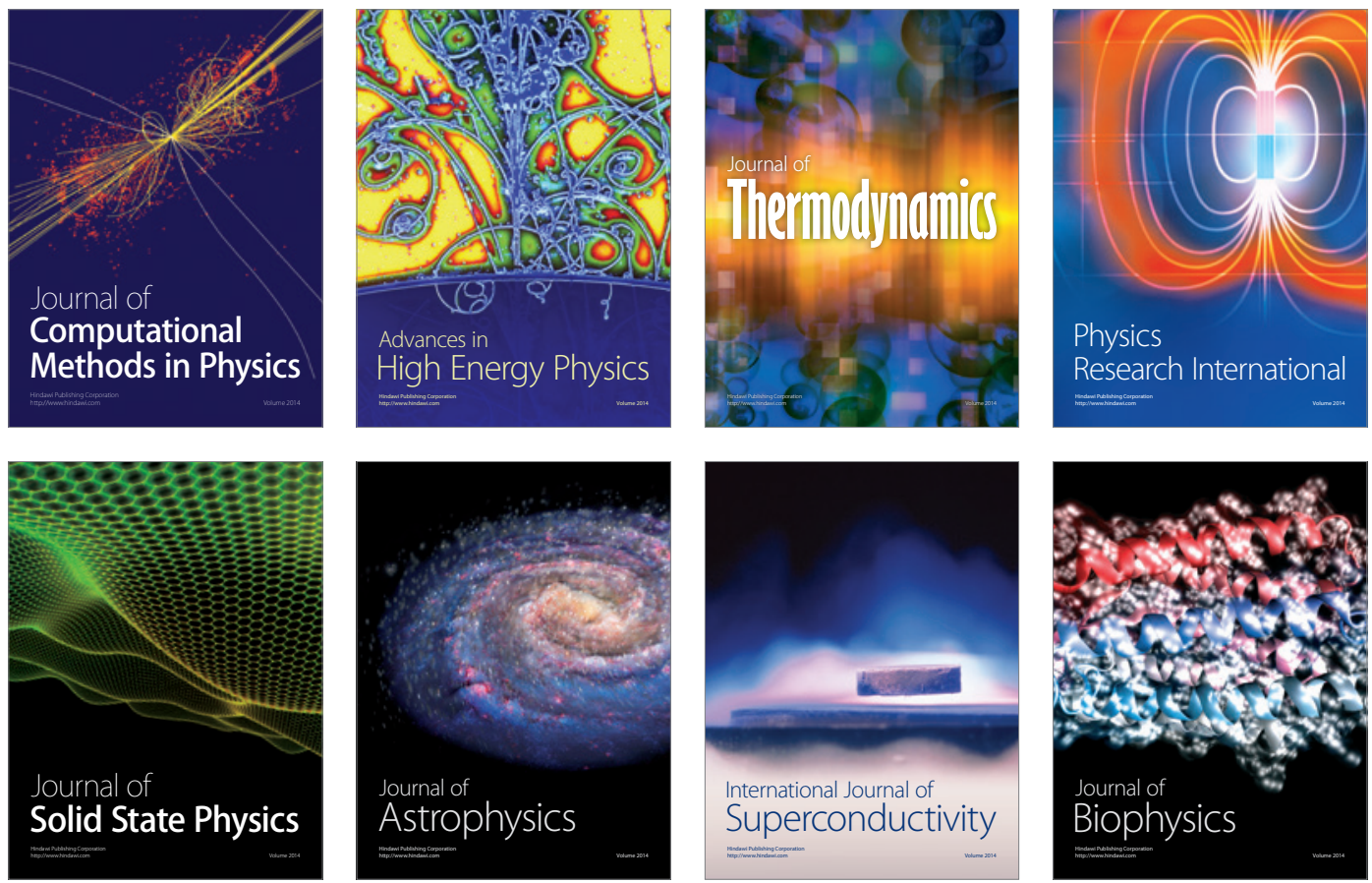
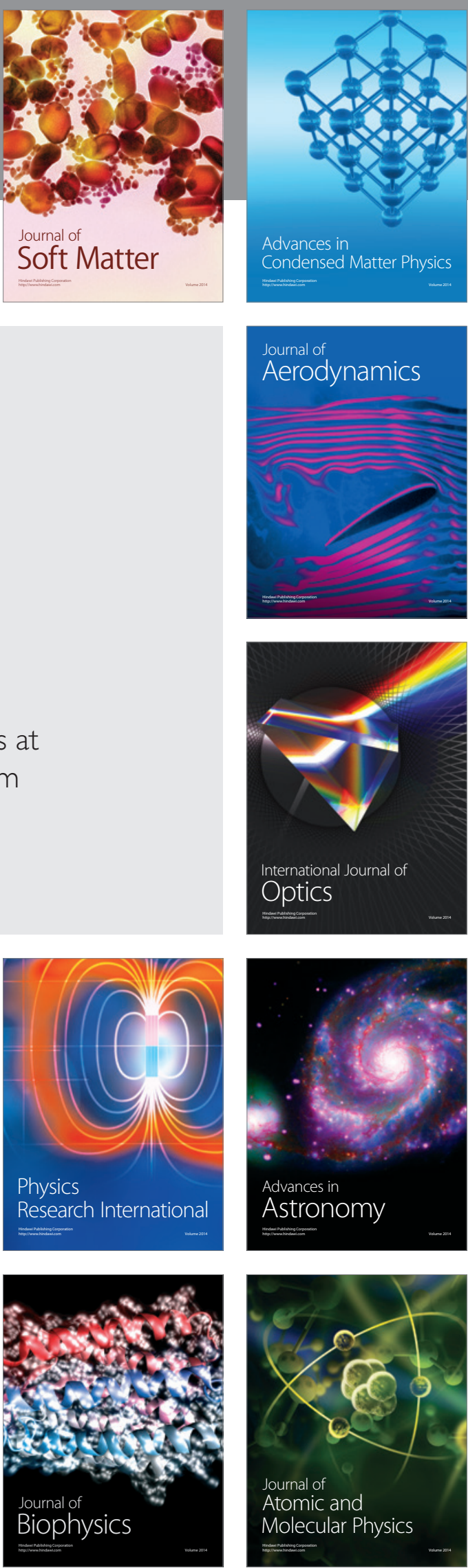